\title{
RESEARCH
}

Open Access

\section{Association of neural tube defects with maternal alterations and genetic polymorphisms in one-carbon metabolic pathway}

Chun-Quan Cai ${ }^{1,2+}$, Yu-Lian Fang ${ }^{3+}$, Jian-Bo Shu ${ }^{4+}$, Lin-Sheng Zhao ${ }^{5+}{ }^{+}$Rui-Ping Zhang ${ }^{6}$, Li-Rong Cao ${ }^{7}$, Yi-Zheng Wang ${ }^{8}$, Xiu-Fang Zhi ${ }^{9}$, Hua-Lei Cui ${ }^{10}$, Ou-Yan Shi ${ }^{11^{*}}$ (D) and Wei Liu ${ }^{12^{*}}$

\begin{abstract}
Background: Neural tube defects (NTDs) are birth defects of the brain, spine, or spinal cord invoked by the insufficient intake of folic acid in the early stages of pregnancy and have a complex etiology involving both genetic and environmental factors. So the study aimed to explore the association between alterations in maternal onecarbon metabolism and NTDs in the offspring.

Methods: We conducted a case-control study to get a deeper insight into this association, as well as into the role of genetic polymorphisms. Plasma concentrations of folate, homocysteine (Hcy), S-adenosylmethionine (SAM), Sadenosylhomocysteine (SAH) and genotypes and alleles distributions of 52 SNPs in 8 genes were compared for 61 women with NTDs-affected offspring and 61 women with healthy ones.

Results: There were significant differences between groups with regard to plasma folate, SAM, SAH and SAM/SAH levels. Logistic regression results revealed a significant association between maternal plasma folate level and risk of NTDs in the offspring. For MTHFD1 rs2236225 polymorphism, mothers having GA genotype and A allele exhibited an increased risk of NTDs in the offspring (OR $=2.600,95 \% \mathrm{Cl}: 1.227-5.529 ; \mathrm{OR}=1.847,95 \% \mathrm{Cl}$ : 1.047-3.259). For MTHFR rs 1801133 polymorphism, mothers having $\Pi$ and $C T$ genotypes were more likely to affect NTDs in the offspring (OR $=4.105,95 \% \mathrm{Cl}: 1.271-13.258 ; \mathrm{OR}=3.333,95 \% \mathrm{Cl}$ : 1.068-10.400). Moreover, mothers carrying $\mathrm{T}$ allele had a higher risk of NTDs in the offspring (OR $=1.798,95 \% \mathrm{Cl}$ : 1.070-3.021). For MTRR rs 1801394 polymorphism, the frequency of $\mathrm{G}$ allele was significantly higher in cases than in controls ( $\mathrm{OR}=1.763,95 \% \mathrm{Cl}$ : 1.023-3.036). Mothers with NTDs-affected children had higher AG genotype in RFC1 rs1051226 polymorphism than controls, manifesting an increased risk for NTDs (OR $=3.923,95 \% \mathrm{Cl}$ : 1.361-11.308).
\end{abstract}

Conclusion: Folic acid deficiency, MTHFD1 rs2236225, MTHFR rs1801133, MTRR rs1801349 and RFC1 rs1051226 polymorphisms may be maternal risk factors of NTDs.

Keywords: Neural tube defects, One-carbon metabolism, Gene, Polymorphism

\footnotetext{
* Correspondence: shiouy@tijmu.edu.cn; lance1971@163.com

${ }^{\dagger}$ Chun-Quan Cai, Yu-Lian Fang, Jian-Bo Shu and Lin-Sheng Zhao contributed equally to this work.

${ }^{11}$ School of Basic Medical Sciences, Tianjin Medical University, No.22

Qixiangtai Road, Heping District, Tianjin 300070, China

${ }^{12}$ Department of Pediatrics, Tianjin Children's Hospital, No.238 Longyan Road,

Beichen District, Tianjin 300134, China

Full list of author information is available at the end of the article
}

(c) The Author(s). 2019 Open Access This article is distributed under the terms of the Creative Commons Attribution 4.0 International License (http://creativecommons.org/licenses/by/4.0/), which permits unrestricted use, distribution, and reproduction in any medium, provided you give appropriate credit to the original author(s) and the source, provide a link to the Creative Commons license, and indicate if changes were made. The Creative Commons Public Domain Dedication waiver (http://creativecommons.org/publicdomain/zero/1.0/) applies to the data made available in this article, unless otherwise stated. 


\section{Background}

Neural tube defects (NTDs) are one of the most severe congenital malformations of the central nervous system at birth [1]. NTDs are birth defects of the brain, spine, or spinal cord that occur because of the insufficient intake of folic acid in the early stages of pregnancy. The symptoms of NTDs will vary depending on the specific kind of birth defect. As reported, the average worldwide prevalence is 1 per 1000 living birth [2]. Studies have reported that the prevalence of NTDs exhibit geographical and cultural specifics [3]. The precise etiology is multifactorial mainly involving both environmental and genetic factors. A series of epidemiological studies indicated one possible reason might be the disturbance of one-carbon metabolism pathway [4]. In 1991, Anonymous et al. first clued that periconceptional supplementation of folic acid was able to prevent the recurrence of NTDs [5], which was further confirmed by many other subsequent studies [6]. From these findings, U.S. Public Health Service recommended that all women of childbearing age should be take $400 \mu \mathrm{g}$ folic acid per day from 4 weeks before impregnation through gestation week 12 in order to prevent first occurrence of a NTDs-affected pregnancy [7].
Moreover, a few studies have suggested impaired homocysteine (Hcy) metabolism might be involved in the protective mechanism of folate [8]. Several studies have also revealed that mothers having children with NTDs possess elevated Hcy level [9]. In addition, genetic factors may also invoke NTDs. Many loci in genes involving in the one-carbon metabolism pathway have been found to be associated with the risk of NTDs, such as methylenetetrahydrofolate reductase (MTHFR), betaine-homocysteine methyltransferase (BHMT), methylenetetrahydrofolate dehydrogenase (MTHFD1), methionine synthase (MTR), methionine synthasereductase $(M T R R)$, and reduced folate carrier (RFC1) [10] (Fig. 1).

In this study, we designed a case-control study to investigate the role of maternal alternations and genetic polymorphisms of one-carbon metabolism pathway in the etiology of NTDs. We measured the concentrations of plasma folic acid, Hcy, and the related biological makers. In addition, we genotyped 52 single nucleotide polymorphisms (SNPs) in 8 genes of one-carbon metabolism pathway to investigate the association between genetic polymorphisms and NTDs.

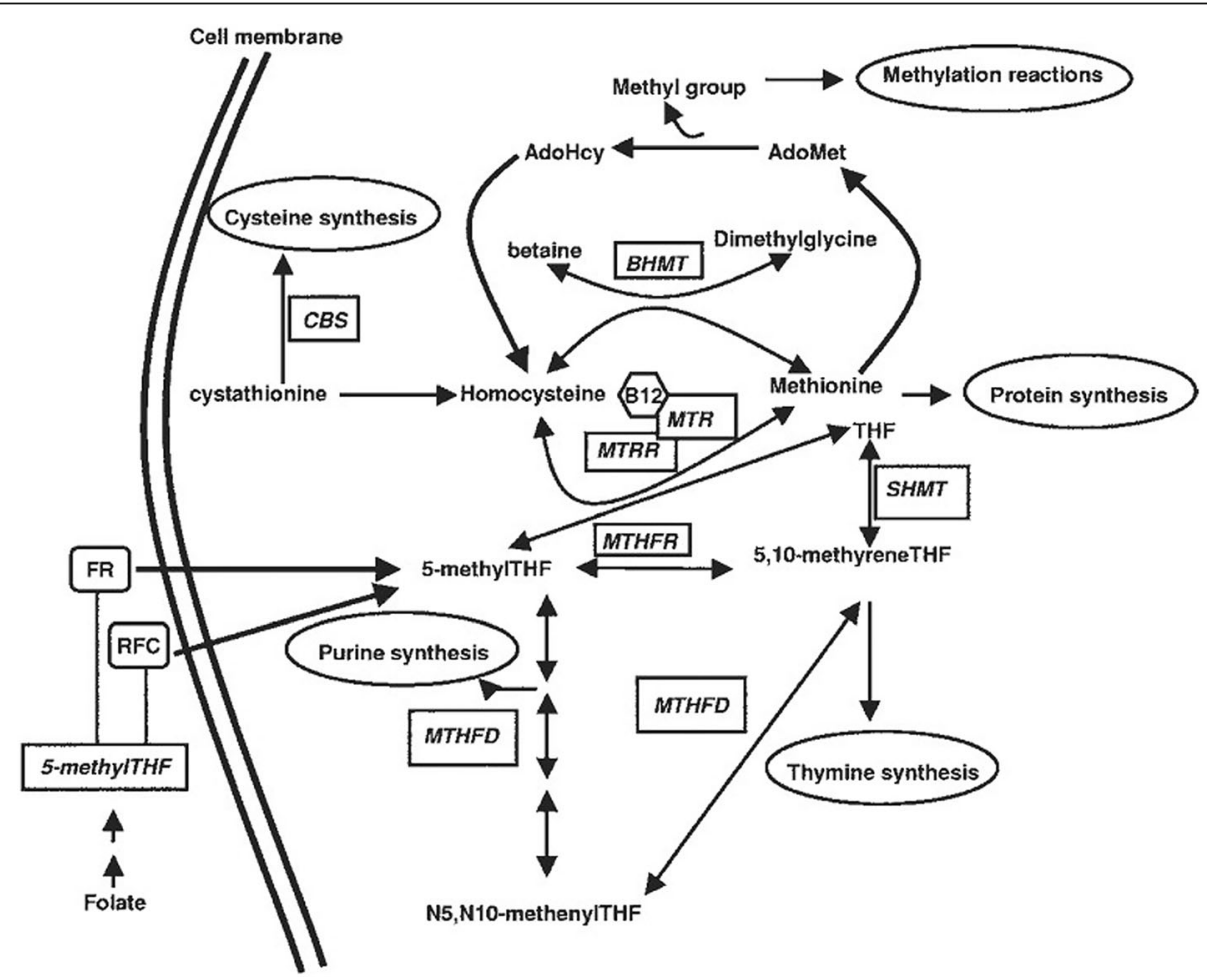

Fig. 1 Simplified overview of one-carbon metabolism pathway. MTHFR, methylene tetrahydrofolate reductase; MTHFD1, methylenetetrahydrofolate dehydrogenase 1; MTR, methionine synthase; MTRR, methionine synthasereductase; RFC1, reduced folate carrier 1; BHMT, betaine-homocysteine methyltransferase; CBS, cystathionine beta synthase;SHMT, serine hydroxymethyltransferase; AdoHcy, Sadenosylhomocysteine; AdoMet, S-adenosylmethionine 


\section{Methods}

\section{Subjects}

The case group consisted of 61 women with NTDs-affected children, which were investigated in the Department of Neurosurgery of Tianjin Children's Hospital in China from November 2010 to May 2014. The diagnosis of NTDs was based on clinical manifestations and images. The control group was composed of 61 age-matched women who had the same ethnic background, but no children affected by NTDs or any other congenital malformations. All participants were from Chinese Han population in the North, Northwest, and Northeast of China. The work was approved by the Tianjin Children's Hospital Ethics Committee and informed consent was obtained from all subjects.

\section{Sample preparation and biomarker measurement}

Peripheral blood samples were collected from all participants in the study after obtaining their consent. The tubes used to collect peripheral blood contained EDTA as anticoagulation. Plasma was separated and stored at $-20^{\circ} \mathrm{C}$. DNA was extracted from blood samples using the DNA Extraction Kit (Tiangen, China) according to the manufacturer instruction. The collected DNA samples were stored at $-80^{\circ} \mathrm{C}$ before use.

Firstly, folic acid concentration was measured using an Access $^{\oplus}$ Immunoassay system (Beckman Coulter, Krefeld, USA). Then, the Hcy concentration was measured using a fluorescence polarization enzyme-based immunoassay (FPIA, Abbott Laboratories, USA). Finally, SAM and $\mathrm{SAH}$ were assayed by the multiple reaction monitoring.

\section{Selection of candidate SNPs}

Eight genes were selected in folate and Hcy metabolism pathway according to relevant references, including BHMT, MTHFD1, MTHFR, MTR, MTRR, RFC1, SHMT1, and thymidylate synthase (TYMS). SNPs were searched and selected using the Haploview4.2 software in HapMap database according to the following criteria: Han population of the Northern China; HWpval ( $P$ value of Hardy-Weinberg equilibrium test) >0.05; MAF (Minor Allele Frequency) $\geq 0.05$. Subsequently, a further analysis was employed to select proper SNPs using functional prediction Website and dbSNP database, and 52 SNPs from 8 candidate genes were selected.

\section{Genotyping}

Fifty-two SNPs of 8 genes were genotyped via the Sequenom-based Mass ARRAY assay. Then, the data of genotype and allele distributions were incorporated and analyzed via the Filemaker Pro Database, which is a cross-platform relational database application from FileMaker Inc. (https://www.filemaker.com).

\section{Statistical analysis}

All statistical analyses were realized via the SPSS 17.0 software. Measurement data were presented as mean \pm standard deviation values, and comparison among groups was conducted by the $t$ - or rank-sum tests. Frequency and rate values were used to denote count data, while comparison among groups was provided by the Chi-square or Fisher's exact tests. The Hardy-Weinberg equilibrium test was conducted in the control group by the Chi-square tests in order to detect group representation. Statistical significance was accepted at $P \leq 0.05$. Odds ratio (OR), with 95\%-confidence interval, was calculated to estimate the relative risk of different genotype combinations. Online SIFI software (http://sift.jcvi.org) and PolyPhen-2 software (http://genetics.bwh.harvard. edu/pph2) were used to predict the potential functions of these pathogenic mutations.

\section{Results}

Maternal biomarkers and risk of NTDs in the offspring

The biomarkers used for cases and controls are listed in Table 1. As can be observed, plasma folate, SAM concentrations and SAM/SAH ratios were significantly lower in cases than that in controls, and the differences showed statistical significance $(P<0.001)$. The SAH concentration of the cases was significantly higher than that of the controls, and the difference showed a statistical significance $(P<0.001)$. Logistic regression results revealed a significant association between maternal folate level and the risk of NTDs in the offspring (Table 2).

\section{Hardy-Weinberg equilibrium}

The genotype frequencies of the vast majority of SNPs accorded with Hardy-Weinberg equilibrium in the control group $(P>0.05)$, except for the following four loci: BHMT rs7700970, MTHFR rs2066470, MTRR rs1532268, and MTRR rs1802059.

\section{Genotype frequencies of various SNPs in cases and controls}

Because 4 SNPs did not conform to Hardy-Weinberg equilibrium, they were excluded from the statistical

Table 1 Distribution of biological makers of folate metabolism in cases and controls

\begin{tabular}{lllll}
\hline & Case $(n=61)$ & Control $(n=61)$ & t values & $P$ values \\
\hline Plasma folate $(\mathrm{nM})$ & $7.7 \pm 1.7$ & $9.4 \pm 1.6$ & -5.687 & $<0.001^{*}$ \\
Plasma Hcy $(\mu \mathrm{M})$ & $12.3 \pm 3.2$ & $11.3 \pm 2.3$ & 1.982 & 0.050 \\
Plasma SAM(nM) & $49.9 \pm 3.0$ & $52.8 \pm 3.2$ & -5.164 & $<0.001^{*}$ \\
Plasma SAH(nM) & $12.9 \pm 1.7$ & $10.1 \pm 1.9$ & 8.578 & $<0.001^{*}$ \\
SAM/SAH & $3.9 \pm 0.6$ & $5.4 \pm 1.0$ & -10.046 & $<0.001^{*}$ \\
\hline
\end{tabular}

Note:* $P$ value $<0.05$ indicates that these biological makers showed a significant difference between case and control groups 
Table $\mathbf{2}$ The results of logistic regression analyses between case and control groups

\begin{tabular}{llllll}
\hline & $\beta$ & $P$ values & $\operatorname{Exp}(\beta)$ & \multicolumn{2}{c}{$95 \%$ Confidence interval for $\beta$} \\
\cline { 5 - 7 } & & & & Lower bound & Upper bound \\
\hline Plasma folate & 0.837 & $0.000^{*}$ & 2.310 & 1.453 & 3.674 \\
Plasma Hcy & -0.244 & 0.117 & .783 & 0.577 & 1.063 \\
Plasma SAM & 0.183 & 0.543 & 1.201 & 0.666 & 2.165 \\
Plasma SAH & 0.185 & 0.879 & 1.203 & 0.112 & 12.963 \\
SAM/SAH & 3.368 & 0.295 & 29.020 & 0.053 & $15,927.004$ \\
constant & -31.100 & 0.046 & .000 & &
\end{tabular}

Note:* $P$ value $<0.05$ indicates that plasma folate level showed a significant difference between case and control groups

$\beta$ : logistic regression coefficient; $\operatorname{Exp}(\beta) \mathrm{e}^{\beta}$ : odds ratio value

analysis, so that the total number of SNPs analyzed in our study was reduced to 48 . The results showed that 4 SNPs of mothers were associated with the susceptibility of NTDs in the offspring $(P<0.05)$, including MTHFD1 rs2236225, MTHFR rs1801133, MTRR rs1801394, RFC1 rs1051266. The observed frequencies of various genotypes and alleles of MTHFD1 rs2236225 polymorphism are listed in Table 3. The case group had a higher frequency of 1958 GA genotype than the control one, and the difference showed a statistical significance $(\mathrm{OR}=$ 2.600, 95\% CI: $1.227-5.529)$, and so did the frequency of A allele (OR $=1.847,95 \% \mathrm{CI}: 1.047-3.259)$.

In terms of the MTHFR rs1801133 polymorphism, the risk of offspring affected by NTDs from mutated homozygous mothers (677 TT genotype) was significantly higher than that from mothers with $677 \mathrm{CC}$ genotype $(\mathrm{OR}=4.015$, 95\% CI: 1.271-13.258). In case of heterozygous mothers, an increased risk of offspring-NTDs was observed $(\mathrm{OR}=3.333$, 95\% CI: $1.068-10.400)$. A pronounced association was found between the maternal $677 \mathrm{~T}$ allele and NTDs in the offspring (OR $=1.798,95 \%$ CI: 1.070-3.021), as is shown in Table 4.

Table 5 presents the data on MTRR rs1801394 polymorphism. There was a statistical difference between case and control groups with respect to $66 \mathrm{G}$ allele (OR = 1.763, 95\% CI: 1.023-3.036).

For the RFC1 rs1051266 polymorphism, genotype and allele frequencies are presented in Table 6. The case group had a higher frequency of mutated AG genotype than the control one, and the difference showed a statistical significance $(\mathrm{OR}=3.923,95 \% \mathrm{CI}$ : 1.361-11.308).

\section{Bioinformatics analysis}

Mutations were analyzed by PolyPhen-2 online software, which predicted the pathogenic nature of missense mutations. The prediction could define the putative role of missense variants and could assess whether they were probably/possibly damaging or benign [11]. Our results manifested that MTHFR rs1801133 and MTRR rs1801394 mutations were "probably damaging", suggesting their potential involvement with NTDs.

SIFT software was used to analyzed the possibility on the pathogenic properties of mutations by comparing the mutant and normal variants. Sift scores of point mutation were calculated for weighting the mutations as damaging or tolerated [12]. Our results suggested MTHFR rs1801133 and MTRR rs1801394 mutations as "damaging" variants.

\section{Discussion}

NTDs are recognized to have a complex etiology, mainly involving both genetic and environmental factors. Several observations supported the opinion that genetic factors mainly involve the planar cell polarity (PCP) signaling pathway $[13,14]$, folate metabolism pathway [15], and glycometabolism pathway [16]. Nuritional factors is the most common environmental factor influencing susceptibility to disease. Folic acid consumption may help to decrease the effects of the low enzymatic activity of one-carbon metabolism pathway [17]. Related studies have verfied that the process of absorption and biotransformation of folic acid to its active form was saturated at doses in region of $200 \sim 400 \mu \mathrm{g}$ of folic acid. The indicator, erythrocyte folate concentration, has been used to assess longer-term folic acid intake and storage [18]. Recent investigations have demonstrated that erythrocyte folate concentration above $1000 \mathrm{nmol} / \mathrm{l}$ was the level required for optimal NTDs prevention. Subsequent studies have further comfirmed that a period of more than 12 weeks with $400 \mu \mathrm{g}$ per day was needed to achieve the reported level of erythrocyte folate concentration [19]. A number of epidemiological studies have

Table 3 Genotype and allele frequencies of the MTHFD1 G1958A polymorphism in case and control groups

\begin{tabular}{llllll}
\hline Genotype & Case $(\mathrm{N}=61)$ & Control $(\mathrm{N}=61)$ & $X^{2}$ values & OR & $95 \% \mathrm{Cl}$ \\
\hline GG & $23(37.7)$ & $37(60.7)$ & & 1 & $P$ values \\
GA & $34(55.7)$ & $21(34.4)$ & 6.331 & 2.600 & $1.227 \sim 5.529$ \\
AA & $4(6.6)$ & $3(4.9)$ & & 2.145 & $0.440 \sim 10.464$ \\
G & $80(65.6)$ & $95(77.9)$ & & 1 & $0.012^{*}$ \\
A & $42(34.4)$ & $27(22.1)$ & 4.547 & 1.847 & $1.047 \sim 3.259$ \\
\hline
\end{tabular}

Note: ${ }^{*} P$ value $<0.05$ indicates that the genotype and allele distributions of MTHFD1 G1958A polymorphism exhibit a significant difference between case and control groups

${ }^{* *} P$ value are calculated using Fisher's exact test 
Table 4 Genotype and allele frequencies of the MTHFR C677T polymorphism in case and control groups

\begin{tabular}{lllllll}
\hline Genotype & Case $(\mathrm{N}=61)$ & Control $(\mathrm{N}=61)$ & $X^{2}$ values & OR & $95 \% \mathrm{Cl}$ & $P$ values \\
\hline $\mathrm{CC}$ & $5(8.2)$ & $15(24.6)$ & & 1 & 3.333 & $1.068 \sim 10.400$ \\
$\mathrm{CT}$ & $30(49.2)$ & $27(44.3)$ & 4.559 & 4.105 & $1.271 \sim 13.258$ \\
$T$ & $26(42.6)$ & $19(31.1)$ & 5.963 & 1 & $0.033^{*}$ \\
$\mathrm{C}$ & $40(32.8)$ & $57(46.7)$ & & 1.798 & $1.070 \sim 3.021$ & $0.015^{*}$ \\
$T$ & $82(67.2)$ & $65(53.3)$ & 4.945 & $0.026^{*}$ \\
\hline
\end{tabular}

Note: ${ }^{*} P$ value $<0.05$ indicates that the genotype and allele distributions of MTHFR C677T polymorphism exhibit a significant difference between case and control groups

further confirmed that folic acid fortification could prevent NTDs to a large extent $[18,20]$.

The increased SAH was also reported as an effective inhibitor of the cellular methyltransferase activity [21, 22]. Low methionine and SAM concentrations in combination with increased SAH and adenosine concentrations have been reported to be associated with reduced methylation capacity [23]. Our results strongly indicated that mothers with NTDs-affected offspring had higher SAH concentrations, and lower plasma folate and SAM concentrations, as compared to mothers with no such children. Then, logistic regression results revealed a significant association between maternal folate level and the risk of NTDs in the offspring. This implied that decreased folic acid was a maternal risk factors for NTDs in the offspring.

The enzyme activity of MTHFD1 was reported to play a crucial role in one-carbon metabolism by providing folate cofactors for DNA synthesis and cellular methylation reactions [24]. Furthermore, MTHFD1 rs2236225 (G1958A) has been studied in several populations as a functional exonic SNP. A number of studies indicated that MTHFD1 G1958A polymorphism has a significant association with risk of NTDs in both NTDs patient and maternal groups [25]. Our results were consistent with this conclusion, showing a significant association between mothers with AG genotype and A allele, and NTDs in the offspring.

The association between maternal MTHFR gene rs1801133 (C677T) polymorphism and NTDs susceptibility was controversial in different populations worldwide [26, 27]. Our research on NTDs mothers showed a significant association with NTDs in the offspring, suggesting that MTHFR C677T polymorphism was a maternal risk factor with a higher probability for mothers to have NTDs offspring. These data were consistent with those of other researchers who reported a higher prevalence in case mothers as compared to controls [28]. PolyPhen-2 and SIFT softwares prediction supported the result, suggesting the MTHFR C677T as a possibly damaging variant.

Several studies from various populations have indicated that MTRR rs1801394 (A66G) polymorphism was a likely maternal risk factor for development of NTDs pregnancy [29]. Our study revealed a higher risk of NTDs among mothers with G allele, as compared to those with A allele. This implied that MTRR rs1801394 polymorphism was a maternal risk factor for NTDs, which complies with the earlier obtained results. PolyPhen-2 and SIFT softwares prediction supported the result, suggesting the MTRR A66G as a possibly damaging variant.

$R F C-1$ is a vital transporter for the folate substrate 5-methyltetrahydrofolate. This transporter is of particular importance during embryonic development in transporting folate across the placenta [30]. RFC-1 rs1051266 polymorphism (A80G) might impair folate transport from maternal blood to the fetus, which could be a maternal risk factor of NTDs [31, 32]. Our findings confirmed a statistically significant difference between RFC-1 80AG genotype in cases and controls. The maternal $R F C-1$ rs 1051266 polymorphism was considered as a risk factor for NTDs in the offspring.

Table 5 Genotype and allele frequencies of the MTRR A66G polymorphism in case and control groups

\begin{tabular}{|c|c|c|c|c|c|c|}
\hline Genotype & Case $(\mathrm{N}=61)$ & Control(N = 61) & $x^{2}$ values & OR & $95 \% \mathrm{Cl}$ & $P$ values \\
\hline$\overline{\mathrm{AA}}$ & $22(36.1)$ & $33(54.1)$ & & 1 & & \\
\hline$A G$ & $31(50.8)$ & $24(39.3)$ & 2.947 & 1.938 & $0.908 \sim 4.136$ & 0.086 \\
\hline GG & $8(13.1)$ & $4(6.6)$ & 2.833 & 3.000 & $0.805 \sim 11.184$ & 0.092 \\
\hline A & $75(61.5)$ & $90(73.8)$ & & 1 & & \\
\hline$G$ & $47(38.5)$ & $32(26.2)$ & 4.212 & 1.763 & $1.023 \sim 3.036$ & $0.040^{*}$ \\
\hline
\end{tabular}

Note: ${ }^{*} P$ value $<0.05$ indicates that the genotype and allele distributions of MTRR A66G polymorphism exhibit a significant difference between case and control groups 
Table 6 Genotype and allele frequencies of the RFC1 A80G polymorphism in case and control groups

\begin{tabular}{lllllll}
\hline Genotype & Case $(\mathrm{N}=61)$ & Control $(\mathrm{N}=61)$ & $x^{2}$ values & OR & $95 \% \mathrm{Cl}$ & $P$ values \\
\hline AA & $6(9.8)$ & $17(27.9)$ & & 1 & 3.923 & $1.361 \sim 11.308$ \\
AG & $36(59.0)$ & $26(42.6)$ & 6.863 & 2.991 & $0.964 \sim 9.276$ \\
GG & $19(31.1)$ & $18(29.5)$ & 3.725 & 1 & $0.009^{*}$ \\
A & $48(39.3)$ & $60(49.2)$ & & 1.492 & $0.898 \sim 2.479$ \\
G & $74(60.7)$ & $62(50.8)$ & 2.392 & 0.054
\end{tabular}

Note: ${ }^{*} P$ value $<0.05$ indicates that the genotype and allele distributions of $R F C 1$ A80G polymorphism showed significant differences between case and control groups

\section{Conclusions}

Based on the results obtained, a conclusion can be drawn that the maternal folate supplementation can reduce the incidence of NTDs in the offspring, while MTHFD1 rs2236225, MTHFR rs1801133, MTRR rs1801394and RFC-1 rs1051266 polymorphisms may increase the risk of NTDs in Han Chinese population of Northern China. Due to the limited sample size in our study, further investigations with a larger sample size and more functional analysis have to be conducted, in order to get a deeper insight into the etiology of NTDs.

\section{Abbreviations \\ AdoHcy: S-adenosylhomocysteine; AdoMet: S-adenosylmethionine; BHMT: Betaine-homocysteine methyltransferase; CBS: Cystathionine beta synthase; Hcy: Homocysteine; MTHFD1: Methylenetetrahydrofolate dehydrogenase; MTHFR: Methylenetetrahydrofolate reductase; MTR: Methionine synthase; MTRR: Methionine synthasereductase; NTDs: Neural tube defects; RFC1: Reduced folate carrier; SAH: S- adenosylhomocysteine; SAM: S-adenosylmethionine; SHMT: Serine hydroxymethyltransferase; SNPS: Single nucleotide polymorphisms; TYMS: Thymidylate synthase}

\section{Acknowledgements}

We are grateful to all the participants that contributed to this study and to the staff of Tianjin Children's Hospital for their cooperation and support in the collection of samples. We thank the parents who agreed to participate in the study.

\section{Funding}

This work was supported by the National Natural Science Foundation of China (\#81771589), the Program of Tianjin Science and Technology Plan (\# 18ZXDBSY00170), and the Science and Technology Project of Tianjin (\#14RCGFSY00150).

\section{Availability of data and materials}

The dataset and analyses are available from the corresponding author on reasonable request.

\section{Authors' contributions}

C-QC, Y-LF, J-BS and L-SZ analysed data and drafted the manuscript. R-PZ and $L-R C$ were responsible for experimental studies. $Y-Z W$ and $X-F Z$ were responsible for data collection. $\mathrm{H}-\mathrm{LC}$ was responsible for clinical diagnosis $\mathrm{O}-\mathrm{YS}$ and WL participated in the design and coordination of this study in addition to revising and critiquing the manuscript. All authors read and approved the final manuscript.

\section{Ethics approval and consent to participate}

This study was approved by the Tianjin Children' Hospital Ethics Committee. The guardian (parents) of the patient consented to both participation and publication of the case.

\section{Consent for publication}

Informed consent was obtained from the guardian (parents), who agreed to join this study, and using the medical information for scientific research and publication.

\section{Competing interests}

The authors declare that they have no competing interests.

\section{Publisher's Note}

Springer Nature remains neutral with regard to jurisdictional claims in published maps and institutional affiliations.

\section{Author details}

${ }^{1}$ College of Management and Economics, Tianjin University, No.92 Weijin Road, Tianjin 300072, China. 'Department of Neurosurgery, Tianjin Children's Hospital, No.238 Longyan Road, Beichen District, Tianjin 300134, China. ${ }^{3}$ Institute of Pediatrics, Tianjin Children's Hospital, No.238 Longyan Road, Beichen District, Tianjin 300134, China. ${ }^{4}$ Institute of Pediatrics, Tianjin Children's Hospital, No.238 Longyan Road, Beichen District, Tianjin 300134, China. ${ }^{5}$ Department of Pathology, Tianjin Children's Hospital, No.238 Longyan Road, Beichen District, Tianjin 300134, China. ${ }^{6}$ Department of Pediatrics, Tianjin Children's Hospital, No.238 Longyan Road, Beichen District, Tianjin 300134, China. ${ }^{7}$ Graduate School of Tianjin Medical University, No.22 Qixiangtai Road, Heping District, Tianjin 300070, China. ${ }^{8}$ Graduate School of Tianjin Medical University, No.22 Qixiangtai Road, Heping District, Tianjin 300070, China. ${ }^{9}$ Graduate School of Tianjin Medical University, No.22 Qixiangtai Road, Heping District, Tianjin 300070, China. ${ }^{10}$ Department of Surgery, Tianjin Children's Hospital, No.238 Longyan Road, Beichen District, Tianjin 300134, China. ${ }^{11}$ School of Basic Medical Sciences, Tianjin Medical University, No.22 Qixiangtai Road, Heping District, Tianjin 300070, China.

${ }^{12}$ Department of Pediatrics, Tianjin Children's Hospital, No.238 Longyan Road, Beichen District, Tianjin 300134, China.

Received: 10 July 2018 Accepted: 4 March 2019

Published online: 14 March 2019

\section{References}

1. Ouyang S, Liu Z, Li Y, Ma F, Wu J. Cystathionine beta-synthase 844ins68 polymorphism is unrelated to susceptibility to neural tube defects. Gene. 2014:535:119-23.

2. Copp AJ, Greene ND, Murdoch JN. The genetic basis of mammalian neurulation. Nat Rev Genet. 2003:4:784-93.

3. Frey L, Hauser WA. Epidemiology of neural tube defects. Epilepsia. 2003; 44(Suppl 3):4-13.

4. Li K, Wahlqvist ML, Li D. Nutrition, one-carbon metabolism and neural tube defects: a review. Nutrients. 2016:8:741.

5. Anonymous. Prevention of neural tube defects: results of the Medical Research Council vitamin study; MRC vitamin study research group. Lancet. 1991:338(8760):131-7.

6. Czeizel $A E$, Dudas I. Prevention of the first occurrence of neural-tube defects by periconceptional vitamin supplementation. NEJM. 1992;327:1832-5.

7. Centers for Disease Control and Prevention. Recommendations for the use of folic acid to reduce the number of cases of spina bifida and other neural tube defects. MMWRRecomm Rep. 1992;11:1-7. 
8. Dunlevy LP, Chitty LS, Burren KA, Doudney K, Stojilkovic-Mikic T, Stanier P, et al. Abnormal folate metabolism in fetuses affected by neural tube defects. Brain. 130:1043-9.

9. Yang M, Li W, Wan Z, Du Y. Elevated homocysteine levels in mothers with neural tube defects: a systematic review and meta-analysis. J Matern Fetal Neonatal Med. 2017;30:2051-7.

10. Zhang T, Lou J, Zhong R, Wu J, Zou L, Sun Y, et al. Genetic variants in the folate pathway and the risk of neural tube defects: a meta-analysis of the published literature. PLoS One. 2013;8:e59570.

11. Adzhubei IA, Schmidt S, Peshkin L, Ramensky VE, Gerasimova A, Bork P, Kondrashov AS, Sunyaev SR, et al. A method and server for predicting damaging missense mutations. Nat Methods. 2010;7:248-9.

12. Ng PC, Henikoff S. SIFT: predicting amino acid changes that affect protein function. Nucleic Acids Res. 2003;31:3812-4.

13. Cai $\mathrm{C}$, Shi $\mathrm{O}$. Genetic evidence in planar cell polarity signaling pathway in human neural tube defects. Front Med. 2014;8:68-78.

14. Cai C, Shi O, Wang B, Chang B, Yang R, Wang Y, et al. Association between VANGL1 gene polymorphisms and neural tube defects. Neuropeditrics. 2014;45:234-9

15. Fang $Y$, Zhang R, Zhi X, Zhao L, Cao L, Wang Y, et al. Association of main folate metabolic pathway gene polymorphisms with neural tube defects in Han population of northern China. Childs Nerv Syst. 2018;34(4):725-9.

16. Fu Y, Wang LL, Yi D, Jin L, Liu J, Zhang $Y$, et al. Association between maternal single nucleotide polymorphisms in genes regulation glucose metabolism and risk for neural tube defects in offspring. Birth Defects Res A Clin Mol Teratol. 2015;103:471-8.

17. Preventive Services Task Force US, Bibbins-Domingo K, Grossman DC. Folic acid supplementation for the prevention of neural tube defects: US preventive services task force recommendation statement. JAMA. 2017; 317(2):183-9.

18. Centers for Disease Control and Prevention. CDC grand rounds: additional opportunities to prevent neural tube defects with folic acid fortification. MMWR Morb Mortal Wkly Rep. 2010;59(31):980-4.

19. Fischer M, Stronati M, Lanari M. Mediterranean diet, folic acid, and neural tube defects. Ital J Pediatr. 2017;43:74.

20. Mills JL. Strategies for preventing folate-related neural tube defects supplements, fortified foods, or both. JAMA. 2017;317(2):144-5.

21. Finnell RH, Spiegelstein O, Wlodarczyk B, Triplett A, Pogribny IP, Melnyk S, et al. DNA methylation in Folbp1 knockout mice supplemented with folic acid during gestation. J Nutr. 2002;132:2457S-61S.

22. Ehrlich $\mathrm{M}$. Expression of various genes is controlled by DNA methylation during mammalian development. J Cell Biochem. 2003;88:899-910.

23. Yi P, Melnyk S, Pogribna M, Pogribny IP, Hine RJ, James SJ. Increase in plasma homocysteine associated with parallel increases in plasma Sadenosylhomocysteine and lymphocyte DNA hypomethylation. J Biol Chem. 2000;275:29318-23.

24. Parle-McDermott A, Kirke PN, Mills JL, Molloy AM, Cox C, O'Leary VB, et al. Confirmation of the R653Q polymorphism of the trifunctional C1-synthase enzyme as a maternal risk for neural tube defects in the Irish population. Eur J Hum Genet. 2006;14:768-72.

25. Carroll N, Pangilinan F, Molloy AM, Troendle J, Mills JL, Kirke PN, et al. Analysis of the MTHFD1 promoter and risk of neural tube defects. Hum Genet. 2009;125:247-56.

26. Liu Z, Zhang J, Liu D, Hao YH, Chang BM, Xie J, et al. Interaction between maternal 5, 10-methylenetetrahydrofolate reductase C677T and methionine synthase A2756G gene variants to increase the risk of fetal neural tube defects in a Shanxi Han population. Chin Med J. 2013;126:865-9.

27. Lucock M, Daskalakis I, Briggs D, Yates Z, Leveve M. Altered folate metabolism and disposition in mothers affected by a spina bifida pregnancy: influence of $677 \mathrm{c} \rightarrow$ t methylenetetrahydrofolate reductase and $2756 \mathrm{a} \rightarrow \mathrm{g}$ methionine synthase genotypes. Mol Genet Metab. 2000;70:2744.

28. Fang $Y$, Ma S, Shi O, Zhang P, Cai C. Association between maternal MTHFR C677T polymorphism and neural tube defects in offspring: a meta-analysis Tianjin Med J. 2015;43:552-8.

29. Ouyang S, Li Y, Liu Z, Chang H, Wu J. Association between MTR A2756G and MTRR A66G polymorphisms and maternal risk for neural tube defects: a meta-analysis. Gene. 2013;515:308-12.

30. Matherly LH, Goldman ID. Membrane transport of folate. Vitam Horm. 2003; 66:403-56.
31. Cao L, Wang Y, Zhang R, Dong L, Cui H, Fang Y, et al. Association of neural tube defects with gene polymorphisms in one-carbon metabolic pathway. Childs Nerv Syst. 2018;34:277-84.

32. Shang Y, Zhao H, Niu B, Li Wl, Zhou R, Zhang T, et al. Correlation of polymorphism of MTHFRs and RFC- 1 genes with neural tube defects in China. Birth Defects Res A Clin Mol Teratol. 2008:82:3-7.
Ready to submit your research? Choose BMC and benefit from:

- fast, convenient online submission

- thorough peer review by experienced researchers in your field

- rapid publication on acceptance

- support for research data, including large and complex data types

- gold Open Access which fosters wider collaboration and increased citations

- maximum visibility for your research: over $100 \mathrm{M}$ website views per year

At BMC, research is always in progress.

Learn more biomedcentral.com/submissions 